\title{
Communication of the Results of the Treatment with Probiotics in Two Cats with Chronic Gingivostomatitis
}

\author{
Brian Morales Segovia' ${ }^{1,2}$, María de los Ángeles Calvo Torras ${ }^{1,2}$ \\ ${ }^{1}$ Faculty of Veterinary Medicine, Universitat Autònoma de Barcelona (UAB), Cerdanyola del Vallès, Spain \\ ${ }^{2}$ Department of Animal Health and Anatomy, Research Group in Applied Microbiology and Environmental, Barcelona, Spain \\ Email: brianmorales2@hotmail.com
}

How to cite this paper: Segovia, B. and Calvo, M. (2018) Communication of the Results of the Treatment with Probiotics in Two Cats with Chronic Gingivostomatitis. Open Journal of Veterinary Medicine, 8, 9-14.

https://doi.org/10.4236/ojvm.2018.82002

Received: December 13, 2017

Accepted: February 25, 2018

Published: February 28, 2018

Copyright $\odot 2018$ by authors and Scientific Research Publishing Inc. This work is licensed under the Creative Commons Attribution International License (CC BY 4.0).

http://creativecommons.org/licenses/by/4.0/

\begin{abstract}
Feline chronic gingivostomatitis (GECF) is a disease of unknown etiology and considered as one of the most frequent paradontal diseases in cats of medium and/or advanced age. This problem is aggravated in the case of cats suffering from some immunosuppressive pathology such as feline leukemia virus (FeLV), feline immunodeficiency virus (HIV) or feline panleukopenia virus (VPF), in which the probability of suffering from GECF is increased. There is a wide variety of treatments against GECF but none of them ensures a complete cure of the disease, with signs of recurrence being observed at the month of treatment. For these reasons, in this investigation we have evaluated a strain of Lactobacillus plantarum with probiotic capacity for the preventive and/or therapeutic purpose of this oral pathology in cats. This investigation was performed in accordance with the Institutional Animal Ethics Committee of the Veterinary Faculty of the Autonomous University of Barcelona.
\end{abstract}

\section{Keywords}

Feline Chronic Gingivostomatitis, Probiotic, Lactobacillus plantarum, Feline Panleucopenia, Feline Leukemia, Feline Immunodeficiency

\section{Introduction}

Currently a growing number of families adopt cats with some immunosuppressive pathology such as those produced by feline leukemia virus (FeLV), feline immunodeficiency virus (FIV) or feline panleucopenia (VPF) survivors. These animals with a poor immune system are prone to secondary and opportunistic infections such as chronic feline gingivostomatitis. 


\subsection{Feline Immunodeficiency Virus (FIV)}

The mechanisms that give rise to immunodeficiency status in virus infection are not fully understood but a decline in the $\mathrm{CD} 4+$ lymphocyte population is observed and this decrease and suppression of $\mathrm{T}$ cell functions justify the immunodeficiency status and subsequent Infections in cat [1].

\subsection{Feline Leukemia Virus (FeLV)}

In the case of this virus, three types of alterations are observed: neoplastic diseases, bone marrow suppression syndromes and immunodeficiency. This immunodeficiency is due to a drastic decrease in the populations of lymphocytes and granulocytes, which favors the appearance of secondary and opportunistic infections [2].

\subsection{Feline Panleukopenia Virus (VPF).}

It is characterized by sudden onset, rapid course, morbidity of $100 \%$ and high mortality producing leucopenia and enteritis. In the case of surviving cats it may end up with sequelae such as Feline chronic gingivostomatitis (GECF) or other oral pathologies [3].

\subsection{Feline Calicivirus (FCV)}

The prevalence of FCV may range from $50 \%$ to $90 \%$ in large groups and cause up to $67 \%$ mortality in clinically healthy adult cats. FCV is associated with acute oral and/or respiratory disease [4].

\subsection{Feline Chronic Gingivostomatitis (GECF)}

There is a very high probability of leukemic cats, with immunodeficiency or survivors of panleucopenia of having GECF. This oral pathology is considered as one of the most frequent paradontal diseases in cats of medium or advanced age.

GEFC is a disease of unknown and probably multifactorial etiology. The most plausible hypothesis is the production of an inadequate immune response of the oral mucosa versus the chronic stimulation exerted by the different oral antigens. [5].

The most frequent clinical signs are: pain in the oral cavity, halitosis, dysphagia, thalism, anorexia and even changes in the behavior of the cat. The oral examination shows severe inflammation of the oral mucosa of the glosses of the palate, palatine arches, and of the gums.

There is a wide variety of treatments against GECF but none of them ensures a complete cure of the disease, with signs of recurrence being observed at the month of treatment. In this study, the use of probiotics is proposed for the preventive and/or therapeutic purpose in relation to oral disease and in order to reduce pharmacological treatment [5].

\subsection{Probiotics}

The World Health Organization defines probiotic as "living microorganisms that 
administered in adequate concentrations and in a sustained way confer a benefit to the health of the host" (WHO).

Traditionally they have been used in order to improve the health of both humans and animals by regulating the intestinal microbiota. Over time it was observed that they can also stimulate the adaptive immune response for the preventive purpose of respiratory and urogenital infections. It has also been suggested to carry out probiotic studies with the purpose of improving oral health [6] [7].

Some of the hypothetical mechanism of probiotics actions in the oral cavity includes:

- Direct interaction in dental plaque,

- Enmeshing in securing of oral micro-organisms to proteins,

- Agility on plaque evolution and on its complex ecosystem by competing and intervening with bacteria attachments,

- Engaging in metabolism of substrate and yielding of chemicals that inhibit oral bacteria.

Indirect probiotic actions featured are:

- Modulating systemic immune function,

- Effect on local immunity,

- Eventuality on no immunologic defense mechanisms,

- Regulation of mucosal permeability,

- Probiotics functions as antioxidants and also produce antioxidants,

- Hamper plaque induction by neutralizing the free electrons [8].

In vitro studies and clinical trials have accumulated evidence in the recent years on the effect of probiotics, especially Lactobacillus spp., in oral, wound and vaginal infections thought a competition and counteraction of pathogens. However, it is necessary to continue isolating and selecting the probiotics strains and studying their effects in order to recompile results [9].

\section{Material and Methodology}

\subsection{Exploration Animal}

The cats were diagnosed by the veterinary team of the Veterinary Hospital (HCV) of the Autonomous University of Barcelona.

They could see in the cats the ulceration of the gingiva and the alveolar mucosa that originated gingivitis, ulcerative stomatitis and caudal stomatitis even it was observed that the labial mucosa, the soft palate and the glossopalatine arch were also affected.

For this reasons the cats had difficulties eating and washing and it were observed sialorrhea, halitosis and pain in the oral cavity when they try to open their mouths.

\subsection{Preparation of the Probiotic}

The microorganism isolated, identified and evaluated for the Research Group 
Applied and Environmental Microbiology of the Autonomous University of Barcelona for administration as a probiotic comes from the microbiota of mare's milk.

An overnight of a liquid culture of Lactobacillus plantarum was taken in 10 $\mathrm{mL}$ of liquid MRS for $24 \pm 2 \mathrm{~h}$ at $37^{\circ} \mathrm{C} \pm 1^{\circ} \mathrm{C}$. After the incubation time the 10 $\mathrm{mL}$ of MRS was transferred to $1 \mathrm{~L}$ of liquid MRS and allowed to incubate under the same conditions as above.

Next, a dilution bank was made in order to know the concentration of the microorganism and then to centrifuge at 6000rpm for 10 minutes at $37^{\circ} \mathrm{C}$.

In parallel, skim milk was autoclaved to resuspend the pellets obtained from the centrifugation, obtaining a final concentration of the microorganism of the order of $1 \times 10^{8} \mathrm{CFU} / \mathrm{mL}$.

After obtaining the optimum concentration for administration, $1 \mathrm{ml}$ of the suspension was dispensed into previously sterilized lyophilization vials. The vials, for $24 \pm 2 \mathrm{~h}$, were frozen at $-20^{\circ} \mathrm{C} \pm 1^{\circ} \mathrm{C}$ and once the freezing time had elapsed, lyophilization proceeded.

The lyophils were found to possess the appropriate concentration by resuspending the contents of three of the lyophils in $9 \mathrm{~mL}$ of MRS each, dilution pools were made, inoculated in MRS plates, incubated and finally counted.

The vials were stored at $4{ }^{\circ} \mathrm{C} \pm 1{ }^{\circ} \mathrm{C}$ to avoid viability losses [10].

\subsection{Probiotic Administration}

The probiotic was administered to two animals: A leukemic cat and a cat surviving feline panleucopenia, both affected by chronic feline gingivostomatitis.

The probiotic was given once daily mixed with food for two months.

\section{Results}

\section{Probiotic Administration}

The administration of the probiotic to the two immunosuppressed cats affected by gingivostomatitis was an improvement in the time of recurrence.

Approximately two weeks after the administration of the probiotic we could observe the disappearance of most symptoms of chronic feline gingivostomatitis. The ulceration, inflammation and pain of the oral cavity decreased, thalism and halitosis disappeared.

During the administration of the probiotic, signs of recurrence were observed after two months of administration, unlike the two weeks or the month, when they are present when pharmacological treatment is administered.

Neither of the two cats stopped eating during this time and neither showed any changes in eating behavior (decreased grooming, screaming during or before eating).

After two months, signs of thalism in one of the cats and pain in the oral cavity were observed when eating in both cats, at which point it was decided to administer anti-inflammatories and antibiotics. 


\section{Discussion}

After administration of the probiotic, has been observed an increase in time in which the cat does not show clinical signs of oral pathology. This is a favorable result that allows us to propose the continuation with the study of probiotics against Feline chronic gingivostomatitis (GECF) or any other type of paradontal disease.

In addition, it has been possible to isolate a strain of Lactobacillus plantarum from the cat's own oral microbiota, which could be interesting to evaluate this lactic acid bacteria for use as probiotic, since belonging to the host's own microbiota would settle better, Give better results than the strain used in this study.

\section{Conclusions}

Feline chronic gingivostomatitis (GECF) is a paradoxical pathology of unknown and possibly multifactorial etiology, which requires further study of the oral microbiology of the cats that suffer from it, in order to reach the conclusion of whether the microbiota itself stimulates the immune response in an exacerbated way that determines Inflammation of the mucosa.

At the same time, the study of probiotics against this pathology should be continued in order to reduce the frequency of treatments that do not guarantee a complete recovery and which does not benefit the health of the cat itself due to the possible appearance of resistance.

\section{References}

[1] Ayala, I., Talone, T., Castillo, C., Gerardi, G. and Hernández, J. (1998) The Acquired Immunodeficiency Syndrome of the Cat Caused by Feline Immunodeficiency Virus). Veterinary Medicine Files, 30, 5-12.

[2] Arjona, A., González, E.E., Soto, I., Barquero, N. and Lucía, E.G. (2000) Seroepidemiolgical Study of Feline Leukemia and Immunodeficiency in Madrid. Veterinary Medicine, 17, 75-83.

[3] Verde, M. and Andrés, M. (1987) Feline Panleukopenia. Small Animal Veterinary Clinic, 7, 123-132.

[4] Henzel, A., Lovato, L.T. and Weiblen, R. (2015) Epidemiological Status of Felid Herpesvirus Type-1 and Feline Calicivirus Infections in Brazil. Ciência Rural, 45, 1042-1049. https://doi.org/10.1590/0103-8478cr20141202

[5] Castro, J.R., Lloret, A., Planellas, M. and Roura, X. (2011) Retrospective Study of 27 Cases of Feline Chronic Gingivostomatitis. Small Animal Veterinary Clinic. A VEPA Official Review, Spanish Small Animal Veterinary Association, 31, 151-157.

[6] Haukioja, A. (2010) Probiotics and Oral Health. European Journal of Dentistry, 4, 348-355.

[7] Rada, V. and Petr, J. (2001) Enumeration of Bifidobacteria in Animal Intestinal Samples. Veterinarni Medicina Praha, 47, 1-4.

[8] Anusha, R.L., Umar, D., Basheer, B. and Baroudi, K. (2015) The Magic of Magic Bugs in Oral Cavity: Probiotics. Journal of Advanced Pharmaceutical Technology \& Research, 6, 43-47. https://doi.org/10.4103/2231-4040.154526

[9] Vuotto, C., Longo, F. and Donelli, G. (2014) Probiotics to Counteract Bio- 
film-Associated Infections: Promising and Conflicting Data. International Journal of Oral Science, 6, 189-194. https://doi.org/10.1038/ijos.2014.52

[10] Rodríguez González, M. (2009) Isolation and Selection of Strains of Lactobacillus Genus with Probiotic and Immunomodulatory Capacity. Ph.D. Dissertation, Autonomous University of Barcelona, Barcelona. 